\title{
Delineation of radiation therapy target volumes for patients with postoperative glioblastoma: a review
}

This article was published in the following Dove Press journal:

OncoTargets and Therapy

27 May 2016

Number of times this article has been viewed

\section{Fen Zhao ${ }^{1,2, *}$ \\ Minghuan $\mathrm{Li}^{1,2, *}$ \\ Li Kong ${ }^{1,2}$ \\ Guoli Zhang ${ }^{1,2}$ \\ Jinming $\mathrm{Yu}^{1,2}$}

'Department of Radiation Oncology, Shandong Cancer Hospital and Institute, Shandong Academy of Medical Sciences, ${ }^{2}$ Key Laboratory of Radiation Oncology Shandong Province, Jinan, Shandong, People's Republic of China

*These authors contributed equally to this work
Correspondence: Jinming Yu Department of Radiation Oncology, Shandong Cancer Hospital and Institute, 440 Jiyan Road, Jinan 250II7, Shandong, People's Republic of China

Tel +86 531 87984729

Fax +86 53| 87984079

Email chinasdyjm@gmail.com

\begin{abstract}
Glioblastoma is the most aggressive and lethal primary malignancy of the brain, and radiotherapy (RT) is a fundamental part of its treatment. However, the optimal radiation treatment conditions are still a matter of debate, and there is no clear consensus concerning the inclusion of peritumoral edema in the clinical target volume calculation. Target delineation calculations that use postoperative residual tumor and cavity volumes plus $2 \mathrm{~cm}$ margins result in smaller volumes of normal brain receiving high-dose irradiation, compared to calculations that include expanded edema. Smaller RT fields may be more appropriate than larger RT fields, possibly reducing the risk of late neurological deterioration, especially in patients with significant peritumoral edema. This review focuses on the factors influencing target delineation, such as peritumoral edema, failure patterns, and prognostic factors (clinical and pathological characteristics) of patients with glioblastoma. Based on this information, we make three suggestions for radiation oncologists to refer to in daily practice. Further study is necessary to investigate the unresolved problems related to routine clinical application of RT.
\end{abstract}

Keywords: glioblastoma, radiotherapy, target volume, postoperative

\section{Introduction}

Glioblastoma multiforme (GBM) is the most common malignant tumor of the central nervous system, accounting for $12 \%-15 \%$ of all intracranial tumors and $50 \%-60 \%$ of gliomas. ${ }^{1}$ There are $\sim 50,000$ patients with GBM in the US, and $\sim 10,000$ new cases are diagnosed annually. ${ }^{2}$ Worldwide, in developed countries, an estimated 3.5 cases of GBM are diagnosed per 100,000 people each year. ${ }^{3}$ The current standard treatment program for newly diagnosed GBM involves surgical resection, to the extent that it is feasible, followed by radiotherapy (RT) with concurrent adjuvant temozolomide (TMZ) chemotherapy. ${ }^{4}$ In malignant gliomas, RT is long established as a critical component of treatment, and its application has evolved over the past decades. A randomized trial in the 1970s showed that 60 Gy of postoperative whole-brain RT (WBRT) could improve the survival for patients with high-grade glioma (HGG). Subsequently, postoperative RT was established as a standard key treatment strategy for newly diagnosed HGG. ${ }^{5}$ However, multiple studies, including the Brain Tumor Cooperative Group 80-01 randomized trial, compared WBRT with partial-brain irradiation and concluded that there was no advantage of WBRT. ${ }^{6}$ As a result, it became standard to treat HGG with partial-brain RT treatment. The introduction of computerized tomography (CT) and magnetic resonance imaging (MRI) has contributed largely to improve the accuracy of tumor delineation. ${ }^{7}$ The three-dimensional (3D) conformal radiation technique makes partial-brain irradiation for glioma possible and reduces neurotoxicity. ${ }^{8} \mathrm{Co}$-registration of pre- and postoperative MRI with planning CT images is normally used to determine 
the RT treatment volume for GBM. However, the optimal treatment volume for GBM remains a controversial issue and varies among cooperative groups. For example, the guidelines of the Radiation Therapy Oncology Group (RTOG) refer to a two-phase treatment at $60 \mathrm{~Gy}$, where the initial clinical target volume (CTV) typically includes postoperative peritumoral edema plus a $2 \mathrm{~cm}$ margin, followed by a boost field defined as the residual tumor plus a $2 \mathrm{~cm}$ margin (as per RTOG 0525 and RTOG 0825 trials). ${ }^{9}$ Conversely, the European Organization for Research and Treatment of Cancer (EORTC) describes a single-phase treatment pattern with 2-3 cm dosimetric margins around the tumor (as evaluated by MRI), because $80 \%-90 \%$ of treatment failures occur within this margin. ${ }^{4}$ The University of Texas MD Anderson Cancer Center uses a $2 \mathrm{~cm}$ margin around the gross tumor volume (GTV), which consists of the resection cavity and any residual contrast enhancing tumor, but ignoring any edema. ${ }^{10}$ In addition, since 2004, several trials from the New Approaches to Brain Tumor Therapy consortium have used margins as small as $5 \mathrm{~mm}$ to delineate the CTV in the treatment of GBM. ${ }^{11}$

Indeed, the margins of the planned target volume vary quite significantly among institutions. A survey of radiation oncologists in Canada showed that $14 \%$ and $32 \%$ followed guidelines from the EORTC and RTOG, respectively, while 54\% followed center-specific guidelines. Singlephase treatment was reported by $60 \%$ of clinicians, and two-phase or multiphase treatments, by $37 \%$. For clinicians treating in single phase, $61 \%$ treat the surgical cavity and enhancing tumor with a margin, and 33\% treat an area that includes tumor edema in addition to the surgical cavity and enhancing tumor. The margins added to the GTV to generate the planning treatment volume (PTV) also varied widely and included $0.5 \mathrm{~cm}(6 \%), 1 \mathrm{~cm}(6 \%), 1.5 \mathrm{~cm}(25 \%), 2 \mathrm{~cm}$ (56\%), $2.5 \mathrm{~cm}$ (25\%), and $3 \mathrm{~cm}$ (12.5\%), with some respondents selecting more than one standard margin. For clinicians treating in multiple phases, $90 \%$ include peritumoral edema in Phase I of the treatment. In Phase II, respondents reported using total margins (from GTV to PTV) of $1 \mathrm{~cm}(10 \%)$, $2 \mathrm{~cm}(40 \%), 2.5 \mathrm{~cm}(30 \%)$, and $3 \mathrm{~cm}(20 \%){ }^{12}$ The calculation of the treatment volume is based on the understanding of peritumoral edema and patterns of postoperative failure in patients with GBM. We believe that this decision should be made in the context of a comprehensive consideration of clinical and pathological prognostic factors. In this article, we review the progress made on these three aspects and identify problems for further discussion.

\section{Peritumoral edema}

The primary reason for the lack of uniform guidelines relates to the aim of treating all possible microscopic areas of the infiltrating tumor. The rationale for including peritumoral edema is that such areas are believed to contain high concentrations of tumor cells. A study compared the histopathologic distributions of neoplastic cells in GBM with the corresponding CT images and found that the vast majority of the neoplastic tissue was contained within the contrastenhancing and low-density peritumoral areas; however, the CT low-density area was not always identical to the area infiltrated by tumor cells. No tumor cells were found in some areas of low density, whereas, in some instances, normal appearing brain tissue beyond the CT low-density area was also found to contain tumor cells. ${ }^{13}$ Furthermore, Halperin et $\mathrm{al}^{14}$ compared preoperative $\mathrm{CT}$ scans with the postmortem topography of recurrent tumors and found that $9 / 11$ (81.8\%) tumor cells were found beyond the enhancement area plus a $1 \mathrm{~cm}$ margin on CT. Indeed, only treatment plans that covered the contrast-enhancing tumor, the "edema" volume, plus an additional $3 \mathrm{~cm}$ margin would cover the entire histologically identified tumor. Kelly et a ${ }^{15}$ also reported on the correlation between histopathologic and MRI findings for 177 biopsy specimens from 39 patients with glial neoplasms. Pathologic evaluation of biopsy specimens obtained from various locations in the volumes defined by CT and MRI showed that contrast enhancement most often corresponded to tumor tissue without intervening parenchyma, whereas hypodensity corresponded to parenchyma infiltrated by isolated tumor cells, or, in some instances, in low-HGGs, to tumor tissue, or to edema. For the normal T1- and T2-weighted MRI regions that were biopsied, there was a false-negative rate of $69 \%$ and $40 \%$, respectively. ${ }^{15}$ A study conducted by Lu et $\mathrm{al}^{16}$ analyzed peritumoral edema using diffusion-tensor MR imaging. This group divided gliomas associated with peritumoral edema into tumor-infiltrated edema and purely vasogenic edema. A drop in fractional anisotropy against the apparent diffusion coefficient in diffusion tensor imaging enabled them to distinguish tumor-infiltrated edema from vasogenic edema. Further pathological control studies are required to confirm this finding.

The number of cases examined in these studies is relatively small, reducing the statistical power. Despite this, they demonstrate the important finding that tumor cells sometimes infiltrate into the peritumoral edema region. The role of peritumoral edema as a prognostic factor in glioma patients is controversial. Schoenegger et $\mathrm{al}^{17}$ considered peritumoral 
edema on a preoperative MRI to be an independent prognostic factor, in addition to the postoperative Karnofsky performance score (KPS), age, and type of tumor resection. Patients with major edema $(>1 \mathrm{~cm})$ had a significant shorter overall survival (OS) time, compared to patients with minor edema $(<1 \mathrm{~cm})$. Pope et al's ${ }^{18}$ study also established that peritumoral edema, noncontrast-enhancing tumor, satellites, and multifocality were independent prognostic factors for survival in GBM, whereas preoperative tumor size, tumor location, and extent of necrosis had no significant impact on survival. Conversely, Iliadis et a ${ }^{19}$ concluded that there was no correlation between peritumoral edema, patient age, and tumor volume, but there was an association between edema, tumor location, and necrosis. Peritumoral edema volume was influenced by the dose of prescribed corticosteroids, and consequently, it was not a reliable measure of tumor burden and could not be used as a predictor of survival. However, netenhancing tumor volume and tumor necrosis were significant independent predictors of OS. Similarly, in a retrospective study by Ramakrishna et al, ${ }^{20}$ the $\mathrm{T} 1$-weighted gadoliniumenhanced area was regarded as solid tumor burden and the hyperintense area on the T2-weighted images was regarded as isolated tumor cell infiltration. They analyzed the predictive value of abnormal MRI features for the survival of patients with GBM. The result demonstrated that tumor burden and invasion characteristics indicated by the T1-weighted gadolinium-enhanced MRI were significant predictors of patient survival, but the total area of signal intensity abnormalities on the T2-weighted images and the T2/T1 ratio did not correlate with patient outcome. As mentioned earlier, published reports concerning the prognostic significance of peritumoral edema in patients with GBM have not been conclusive or consistent. This may be due to variation in the sample size among studies. In addition, the complex composition of peritumoral edema, which is challenging to identify, might also be a contributing factor toward the inconsistent conclusion.

In summary, for patients with GBM, the vast majority of tumor tissues are contained within the contrast enhancement areas in T1-weighted MRI; tumor cells sometimes, but not always, infiltrate into the peritumoral edema area. Furthermore, the significance of peritumoral edema for the survival of patient with GBM is not clear. Therefore, we believe that when delineating the target volume for RT, regarding the peritumoral edema area as a subclinical lesion may be more appropriate. In addition, the ability to accurately distinguish tumor-infiltrated edema from vasogenic edema composed purely of extracellular water could be helpful for target delineation. It is hoped that advances in radiological techniques will enable this in the future.

\section{Recurrent patterns of postoperative GBM}

Chamberlain ${ }^{21}$ retrospectively analyzed the patterns of radiographic presentation of 80 adult patients with supratentorial GBM at four clinically relevant time points: presentation, first recurrence, second recurrence, and third recurrence. At diagnosis, $87.5 \%(70 / 80), 6.25 \%(5 / 80), 3.75 \%$, and $2.5 \%$ of patients presented with unifocal disease, distant, multifocal, and diffuse MRI defined radiographic patterns, respectively. At first recurrence, following the progression on RT/TMZ and before the initiation of bevacizumab, $80 \%$ of GBMs were local, 7.5\% distant, $6.25 \%$ multifocal (including one with cerebrospinal fluid dissemination), and $6.25 \%$ diffuse. At second recurrence, following the progression on bevacizumab, $71.25 \%$ were local, $8.75 \%$ distant, $8.75 \%$ multifocal (2/7 with cerebrospinal fluid dissemination), and $11.25 \%$ diffuse. At third recurrence (57 patients total), $71.25 \%$ were local, $7.0 \%$ distant, $7.0 \%$ multifocal, and $14.0 \%$ diffuse. The majority of adult patients with GBM present with MRI-defined local disease and maintain this pattern notwithstanding multiple recurrences and treatment with bevacizumab. Similarly, Wallner et $\mathrm{a}^{22}$ found that $78 \%$ of unifocal anaplastic astrocytoma and GBM recurrences occurred within $2 \mathrm{~cm}$ of the presurgical original tumor extent, which is defined as the enhancing edge of the tumor on preoperative CT, and $56 \%(18 / 32)$ of tumors recurred within $1 \mathrm{~cm}$ of the initial tumor margin. Extensive preoperative edema was associated with a decreased distance between the initial and recurrent tumor margins. Large tumors were generally not more likely to recur further from the initial tumor margin than were smaller tumors. Subsequently, Liang et al ${ }^{23}$ published the pattern of failure for 42 patients with grade III or IV astrocytoma treated with chemoradiotherapy to a total of $60 \mathrm{~Gy}$. In all 42 patients, recurrence occurred within a $2 \mathrm{~cm}$ margin of the original CT-enhancing lesion, and $10 \%$ of patients suffered from multifocal recurrence. In a retrospective series of 34 patients treated either with WBRT and conformal boost or entirely with 3D conformal RT, Oppitz et $\mathrm{al}^{24}$ revealed that all GBM recurrences occurred within the $90 \%$ isodose line when targets were contoured around the original preoperative contrast-enhancing tumor plus a $2 \mathrm{~cm}$ margin. In a conformal dose-escalation study, Lee et $\mathrm{al}^{25}$ analyzed 36 patients with $\mathrm{HGGs}$ treated with radiation alone 
to $70-80$ Gy using the 3D conformal technique. In this study, recurrences were divided into several categories: 1) "central", in which $95 \%$ or more of the recurrent tumor volume (Vrecur) was within D95, the region treated to a high dose $(95 \%$ of the prescription dose); 2) "infield", in which $80 \%$ or more of Vrecur was within the D95 isodose surface; 3) "marginal", when between $20 \%$ and $80 \%$ of Vrecur was inside the D95 surface; 4) "outwith", in which $<20 \%$ of Vrecur was inside the D95 surface. They found that $89 \%$ of the recurrences featured a central or infield recurrence pattern, 3/36 (8\%) had a marginal recurrence pattern, and only one patient (3\%) clearly failed outside of the high-dose region. More recently, Chang et $\mathrm{al}^{10}$ reported similar patterns of failure in a series of 48 patients with GBM, when comparing treatment plans based on residual tumor and cavity plus $2 \mathrm{~cm}$ margin, as used at the MD Anderson Cancer Center, with plans created according to the RTOG guidelines that specified the inclusion of preoperative peritumoral edema. They showed that $90 \%$ (43/48) of patients failed in central and infield locations. The five remaining marginal and distal recurrences failed to be covered by the 46 Gy isodose line, even when overlaid by the RTOG plan incorporating edema volume, confirming them to be true marginal recurrences. Furthermore, Minniti et $\mathrm{al}^{26}$ compared recurrence patterns in 105 patients whose surgical resections were delineated by the EORTC contouring technique, wherein the CTV includes the resection cavity, any residual tumor seen on postoperative T1-weighted MRI, plus a $2 \mathrm{~cm}$ margin, and the PTV includes the CTV plus an additional $3 \mathrm{~mm}$ margin. After recurrence was confirmed, a theoretical plan, based on the addition of postoperative edema plus $2 \mathrm{~cm}$ margins, according to the current RTOG guidelines, was created for each patient. The radiation coverage of the site of subsequent recurrences was compared for the different contouring techniques. The results revealed no significant differences in relapse patterns between the two target delineation techniques. However, dosimetric analysis showed that the median percent volume of normal brain irradiated to high doses was significantly smaller using the EORTC contouring technique. In our opinion, these data provide some evidence and reassurance to support treatment plans based on resection cavity and any residual tumor seen on postoperative T1-weighted MRI with a $2 \mathrm{~cm}$ margin, rather than specified inclusion of preoperative peritumoral edema plus a $2 \mathrm{~cm}$ margin. The use of this limited-margin RT can significantly decrease the volume of normal brain tissue that is irradiated, without a significant increase in the risk of marginal recurrences. A number of studies have been conducted to explore the feasibility of limited-margin RT in the context of a treatment paradigm involving RT with concurrent chemotherapy. McDonald et $\mathrm{al}^{11}$ analyzed the pattern of tumor failure in a series of 62 patients with GBM treated with postoperative limited-margin RT and concurrent chemotherapy. The initial CTV included the postoperative T2 abnormality, with a median margin of $0.7 \mathrm{~cm}$. The boost CTV included the residual T1-enhancing tumor and resection cavity, with a median margin of $0.5 \mathrm{~cm}$. The PTV margin varied from an additional $0.3 \mathrm{~cm}$ to an additional $0.5 \mathrm{~cm}$. The initial dose was 46-54 Gy, followed by a boost to $60 \mathrm{~Gy}$. In this study, the total boost PTV $\left(\mathrm{PTV}_{\text {boost }}\right)$ margin was $1 \mathrm{~cm}$ or less in $92 \%$ of patients. Within the follow-up time, radiographic tumor progression developed in 43 of 62 patients. Imaging was available for analysis in 41 patients: $38(93 \%)$ had a central or infield failure, two $(5 \%)$ had a marginal failure, and one $(2 \%)$ had a distant failure, relative to the 60 Gy isodose line. The author concluded that a PTV boost margin of $1 \mathrm{~cm}$ or less did not appear to increase the risk of marginal and/or distant tumor failure, compared with other published series. Dobelbower et $\mathrm{al}^{27}$ analyzed the patterns of failure in patients with GBM treated with concurrent radiation and TMZ. Patients generally received 46 Gy to the primary tumor, surrounding edema plus a $1 \mathrm{~cm}$ margin, and $60 \mathrm{~Gy}$ to the enhancing tumor plus a $1 \mathrm{~cm}$ margin. The result revealed that all patients had some forms of failure at the primary site, whether infield or marginal: 18 patients $(90 \%)$ had infield failures, two patients (10\%) had marginal failures, and no regional failures were reported. Four patients (20\%) suffered from distant failure, in which an independent satellite lesion was located completely outwith the $95 \%$ isodose curve. These studies also suggested that by delineating the GTV based on peritumoral edema, it is feasible to reduce the margin to $1 \mathrm{~cm}$ or less. Although clinical data in adults are sparse, the volume of irradiated brain is believed to be an important factor in the development of neurotoxicity and for the development of radiographic and pathologic surrogates for neurotoxicity. ${ }^{28-31}$

Above all, smaller RT fields may be more appropriate than larger RT fields, possibly reducing the risk of late neurological deterioration especially in patients with large peritumoral edema. In long-term survivors, the neurocognitive function would be likely to be affected by radiation therapy, given the adverse effects of radiation on neurogenesis of the hippocampus. ${ }^{32}$ So when contouring the radiation target volume for postoperative GBM, hippocampal sparing is suggested for some patients with tumor adjacent to the hippocampus. The pattern of failure for GBM after radiation therapy has been studied previously; almost all tumors fail 
within a $2 \mathrm{~cm}$ margin of the resection cavity or residual tumor. The primary failure location was infield, but some patients had marginal failures, and few had a distant failure or an independent satellite lesion. Taking these data into consideration, we conclude that it is preferable to contour the GTV based on the T1-enhanced MRI, and regard the peritumoral edema as a subclinical lesion. We suggest that the CTV should be identified based on the residual T1-enhancing tumor and resection cavity (GTV) with a $2 \mathrm{~cm}$ margin, or the postoperative T2 or FLAIR (Fluid-attenuated inversion recovery) abnormality; however, in the case of a cone-down boost phase, the CTV should include the GTV with a $1 \mathrm{~cm}$ margin.

\section{Prognostic factors}

The biological behavior of GBM for individual patients is different, resulting in different treatment responses to RT and chemotherapy. When contouring the RT target volume, this differing biological behavior should be taken into consideration. A number of prognostic factors are now known to correlate with the clinical outcomes ${ }^{33,34}$ of patients with GBM. Several studies have identified clinical-, tumor-, and treatment-related prognostic factors that influence outcomes. ${ }^{35,36}$ The most relevant factors were KPS, age at presentation, extent of surgical resection, postoperative radiation treatment, and histopathological factors..$^{37,38}$

\section{Clinical features}

Previous studies ${ }^{34,39,40}$ have revealed a significant positive relationship between KPS and duration of survival; significantly different outcomes were reported for patients depending on whether their KPS at presentation was greater or less than 70. Advanced age at presentation also correlates with a poor outcome. In most studies, patients younger than 50 years are considered to be young patients, and patients older than this may have poorer survival rates. Korshunov et $\mathrm{al}^{41}$ reported that the percentage of patients younger than 40 years who survived for $>5$ years was $34 \%$, compared with $6 \%$ for patients aged 40 years and older. Consequently, this group suggested that 40 years is the most appropriate cutoff for dividing patients with GBM into age groups according to prognosis. Using recursive-partitioning analysis, Simpson et $\mathrm{al}^{42}$ identified four risk groups. The two lower-risk groups included patients younger than 40 years, with the lowest risk group being young patients with a tumor restricted to the frontal lobe. An intermediate-risk group included patients with KPS $>70$, with subtotal or total resection, and aged between 40 years and 65 years. The highest-risk group included all patients older than 65 years, and patients aged between 40 years and 65 years, with either KPS $<80$ or biopsy only.

\section{Histopathological characteristics}

Histologic features are a major independent prognostic factor for glioma patients, which are to be considered alongside patient age and performance status. ${ }^{43}$ One of the main bases for glioma grading is the proliferation index, which is an independent predictor of survival for GBM. Ki-67 is an excellent proliferation marker that can be used to determine the growth fraction of a given cell population. Immunohistological determination of a high Ki-67 index is strongly associated with shorter OS and shorter progress-free survival. ${ }^{44}$ The cell and tissue composition of the tumor also influences the survival of patients with GBM: fibrocystic changes, calcification, and astrocytic differentiation within the tumor are predictors of a better outcome, whereas necrosis is associated with a worse prognosis. ${ }^{45}$ Numerous studies have reported that $\mathrm{O}^{6}$-methylguanine-DNA-methyltransferase (MGMT) promoter methylation correlates with improved survival in patients with glioma treated with alkylating agents. ${ }^{46,47}$ In a study conducted by Dunn et al, ${ }^{46}$ the MGMT promoters of 58 of 109 cases $(53.2 \%)$ were found to be significantly methylated (defined as methylation $\geq 9 \%$ ). MGMT promoter methylation was highly significantly associated with prolonged progress-free survival and OS. The median OS was 16.8 months with a 2 -year survival of $35.2 \%$ for methylated cases, compared with 11.1 months and $0 \%$ for unmethylated cases. Further studies suggested that the extent of methylation also correlates with survival. Patients with the highest level of methylation ( $>35 \%$ ) had the longest OS (median: 26.2 months) with a 2-year survival of $59.7 \%$. The 2-year survival of patients with medium $(>20 \%$ to $\leq 35 \%)$ and low methylation levels ( $\geq 9 \%$ to $\leq 20 \%$ ) was $34.2 \%$ and $13.3 \%$, respectively. Hegi et $\mathrm{al}^{47}$ reported that of 206 evaluated tumors, 92 (44.7\%) featured detectable MGMT promoter methylation. In the chemoradiotherapy (TMZ plus RT) group, the 2-year survival rate was $46 \%$ among those with a methylated MGMT promoter, and $13.8 \%$ among those with an unmethylated MGMT promoter. In the RT group, the 2-year survival rates of patients with or without promoter methylation were $22.7 \%$ and $<2 \%$, respectively.

The clinical and pathological features of GBM significantly influence the treatment choice, because in the absence of effective treatment, the biological behavior of the tumor typically determines the prognosis. As stated earlier, patients without promoter methylation, despite undergoing the standard treatment, still have poor 2-year survival rates. 
Interestingly, patterns of recurrence were significantly different depending on the methylation status of the MGMT promoter. ${ }^{26}$ More distant recurrences were observed in patients with methylated promoters (31\% vs 5.4\%), while more central/infield recurrences were observed in patients with unmethylated promoters (91\% vs 64\%). This indicates that patients without promoter methylation are more chemoradioresistant. For these patients, further research is warranted to establish whether it would be beneficial to increase the radiation dose or use nonconventional fractionation $\mathrm{RT}$ to improve local control. When creating a RT plan for patients with GBM, it is necessary to refer to the patients' clinical and pathological characteristics to develop an individualized rational target volume.

In conclusion, the main points about peritumoral edema and histopathological characteristics in target volume design are concluded in Table 1. Based on the data described in this review, we also make the following suggestions for radiation oncologists to refer to and to discuss in daily practice, as well as in future clinical trials. They could decide the concrete proposal based on case-by-case basis:

- Patients with a KPS of $\geq 70$, better neurological function, good prognostic factors, and who undergo subtotal or gross total resection could be considered to receive a more aggressive approach, and the treatment target volume could include the peritumoral edema, with a margin (the two-phase model, Figure 1A and B). These patients may gain greater benefit from postoperative chemotherapy and radiation. However, large brain volumes irradiated to high doses especially in the case of significant edema may increase the potential toxicity of radiation treatment.

- Patients who are older (ie, $\geq 70$ years of age), have a KPS of $<70$, or have an unmethylated MGMT promoter tolerate chemoradiotherapy poorly or are chemoradioresistant. These patients are also suggested to be treated with limited-margin RT (Figure 1C).

- Patients with GBM who have undergone only biopsy or partial resection with a large residual tumor, especially in the presence of an unmethylated MGMT promoter, are likely the most appropriate candidates for limited-margin RT. For such patients, the most common failure pattern is expected to be local relapse. The treatment target volume could only include the residual T1-enhancing tumor and the resection cavity with a margin, rather than the inclusion of peritumoral edema.

\section{Conclusion}

Tumor recurrence in patients with GBM treated with RT plus concomitant and adjuvant TMZ predominantly occurs in central and infield localizations, depending on the different target volume delineations. There is no clear correlation between the inclusion of edema and recurrence patterns. At present, treatment planning tends to include the contrast-enhancing tumor on CT/T1-weighted MRI plus a $2 \mathrm{~cm}$ margin, or the FLAIR/T2-weighted abnormality on the postoperative MRI scan plus a $1 \mathrm{~cm}$ margin. No randomized controlled trial has yet been conducted to compare the outcomes between these two target volume delineations directly, to identify which

Table I Conclusions of some studies referenced

\begin{tabular}{|c|c|}
\hline References & Conclusion \\
\hline Burger et al; ${ }^{13}$ Halperin et al ${ }^{14}$ & $\begin{array}{l}\text { The distribution of cells of a GBM cannot be inferred from CT images, since the peritumoral area of low density } \\
\text { can over- or underestimate the extent of the lesion }\end{array}$ \\
\hline Kelly et al ${ }^{15}$ & $\begin{array}{l}\text { For the normal TI- and T2-weighted MRI regions that were biopsied, there was a false-negative rate of } 69 \% \text { and } \\
40 \% \text {, respectively }\end{array}$ \\
\hline Schoenegger et al; ${ }^{17}$ Pope et al ${ }^{18}$ & Peritumoral edema on a preoperative MRI to be an independent prognostic factor \\
\hline Iliadis et al; ${ }^{19}$ & Peritumoral edema was not a reliable measure of tumor burden and could not be used as a predictor of survival. \\
\hline Ramakrishna et $\mathrm{al}^{20}$ & However, net-enhancing tumor volume and tumor necrosis were significant independent predictors of OS \\
\hline $\begin{array}{l}\text { Chamberlain; }{ }^{21} \text { Wallner et al; }{ }^{22} \\
\text { Liang et al; }{ }^{23} \text { Oppitz et } \mathrm{al}^{24}\end{array}$ & $\begin{array}{l}\text { The majority of adult patients with GBM present with MRI-defined local recurrences notwithstanding multiple } \\
\text { recurrences }\end{array}$ \\
\hline Chang et al; ${ }^{10}$ Minniti et al; ${ }^{26}$ & There were no significant differences in relapse patterns between the two target delineation techniques (limited \\
\hline McDonald et al;" & margin based on resection cavity and any residual tumor seen on postoperative TI-weighted MRI with a $2 \mathrm{~cm}$ \\
\hline Dobelbower et $\mathrm{al}^{27}$ & $\begin{array}{l}\text { margin vs inclusion of preoperative peritumoral edema plus a } 2 \mathrm{~cm} \text { margin). The use of this limited-margin RT } \\
\text { can significantly decrease the volume of normal brain tissue that is irradiated }\end{array}$ \\
\hline $\begin{array}{l}\text { Siker et } \mathrm{al}^{34} \text { Scott et } \mathrm{al} ;{ }^{39} \\
\text { Ulutin et al; }{ }^{40} \text { Korshunov et } \mathrm{al}^{41}\end{array}$ & $\begin{array}{l}\text { There is a significant positive relationship between KPS, age, and duration of survival; patients with higher KPS } \\
\text { and younger age have longer survival }\end{array}$ \\
\hline Preusser et al; $^{44}$ Biernat; ${ }^{45}$ & Histologic features such as proliferation index $\mathrm{Ki}-67$, cell and tissue composition of the tumor, and MGMT \\
\hline \multirow[t]{2}{*}{ Dunn et al; ${ }^{46}$ Minniti et al ${ }^{26}$} & promoter methylation are major independent prognostic factors for patients with glioma. \\
\hline & Patterns of recurrence were significantly different depending on the methylation status of the MGMT promoter \\
\hline
\end{tabular}

Abbreviations: CT, computerized tomography; GBM, glioblastoma multiforme; KPS, Karnofsky performance score; MGMT, O'-methylguanine-DNA-methyltransferase; MRI, magnetic resonance imaging; OS, overall survival; RT, radiotherapy. 

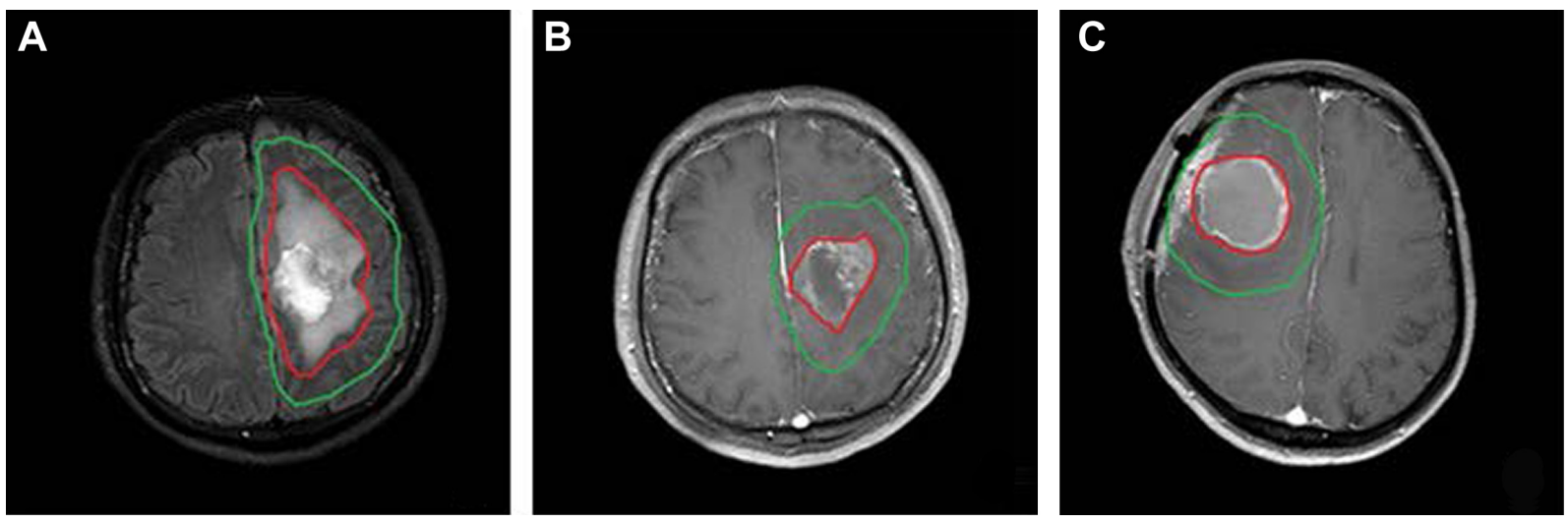

Figure I Target delineation for GBM.

Notes: Figures $\mathbf{A}$ and $\mathbf{B}$ are MRI images from a 58 year old male patient with GBM with a large residual tumor, who had undergone partial resection. These figures show the two phases of target volume delineation. (A) The initial GTV includes postoperative peritumoral edema based on the axial T2 fluid-attenuated inversion recovery sequence (red line); the initial CTV (green line) includes postoperative peritumoral edema plus a $2 \mathrm{~cm}$ expansion in all directions. (B) The boost GTV includes the surgical cavity and residual enhancement based on the axial TI sequence with gadolinium (red line) and the boost CTV is a $2 \mathrm{~cm}$ expansion in all directions (green line). Figure $\mathbf{C}$ is a MRI image from a 39 year old female patient with GBM, who had undergone gross total resection. It shows the one phase of target volume delineation. The GTV includes surgical cavity and residual enhancement based on the axial TI sequence with gadolinium (red line) and CTV is a $2 \mathrm{~cm}$ expansion in all directions (green line).

Abbreviations: CTV, clinical target volume; GBM, glioblastoma multiforme; GTV, gross target volume; MRI, magnetic resonance imaging.

is optimal. The PTV is generally generated from the CTV with less than a $1 \mathrm{~cm}$ margin. The biological behavior of the tumor, which potentially determines the prognosis and the treatment, also has an obvious impact on survival. Nowadays, new techniques of RT administration have evolved to improve target coverage with escalating doses and to spare unaffected organs in close vicinity by means of steep dose gradients. High-precision radiation therapy necessitates precise target delineation. Consequently, when creating the RT plan for a patient with GBM, it is necessary to refer to the patients' clinical and pathological characteristics and design an individualized target volume. Future studies are also required to create a risk model for recurrence estimation to provide radiation oncologists with quantifiable information and facilitate decision making.

\section{Disclosure}

The authors report no conflicts of interest in this work.

\section{References}

1. Hess KR, Broglio KR, Bondy ML. Adult glioma incidence trends in the United States, 1977-2000. Cancer. 2004;101(10):2293-2299.

2. Porter KR, McCarthy BJ, Freels S, Kim Y, Davis FG. Prevalence estimates for primary brain tumors in the United States by age, gender, behavior, and histology. Neuro Oncol. 2010;12(6):520-527.

3. Ohgaki H, Kleihues P. Population-based studies on incidence, survival rates, and genetic alterations in astrocytic and oligodendroglial gliomas. J Neuropathol Exp Neurol. 2005;64(6):479-489.

4. Stupp R, Mason WP, van den Bent MJ, et al. Radiotherapy plus concomitant and adjuvant temozolomide for glioblastoma. $N$ Engl J Med. 2005;352(10):987-996.

5. Walker MD, Alexander E Jr, Hunt WE, et al. Evaluation of BCNU and/ or radiotherapy in the treatment of anaplastic gliomas. A cooperative clinical trial. J Neurosurg. 1978;49(3):333-343.
6. Shapiro WR, Green SB, Burger PC, et al. Randomized trial of three chemotherapy regimens and two radiotherapy regimens and two radiotherapy regimens in postoperative treatment of malignant glioma. Brain Tumor Cooperative Group Trial 8001. J Neurosurg. 1989;71(1):1-9.

7. Heesters MA, Wijrdeman HK, Struikmans H, Witkamp T, Moerland MA. Brain tumor delineation based on CT and MR imaging. Implications for radiotherapy treatment planning. Strahlenther Onkol. 1993; 169(12):729-733.

8. Leibel SA, Scott CB, Loeffler JS. Contemporary approaches to the treatment of malignant gliomas with radiation therapy. Semin Oncol. 1994; 21(2):198-219.

9. Colman H, Berkey BA, Maor MH, et al. Phase II Radiation Therapy Oncology Group trial of conventional radiation therapy followed by treatment with recombinant interferon-beta for supratentorial glioblastoma: results of RTOG 9710. Int J Radiat Oncol Biol Phys. 2006;66(3): $818-824$.

10. Chang EL, Akyurek S, Avalos T, et al. Evaluation of peritumoral edema in the delineation of radiotherapy clinical target volumes for glioblastoma. Int J Radiat Oncol Biol Phys. 2007;68(1):144-150.

11. McDonald MW, Shu HK, Curran WJ Jr, Crocker IR. Pattern of failure after limited margin radiotherapy and temozolomide for glioblastoma. Int J Radiat Oncol Biol Phys. 2011;79(1):130-136.

12. Ghose A, Lim G, Husain S. Treatment for glioblastoma multiforme: current guidelines and Canadian practice. Curr Oncol. 2010;17(6):52-58.

13. Burger PC, Dubois PJ, Schold SC Jr, et al. Computerized tomographic and pathologic studies of the untreated, quiescent, and recurrent glioblastoma multiforme. J Neurosurg. 1983;58(2):159-169.

14. Halperin EC, Bentel G, Heinz ER, Burger PC. Radiation therapy treatment planning in supratentorial glioblastoma multiforme: an analysis based on post mortem topographic anatomy with CT correlations. Int J Radiat Oncol Biol Phys. 1989;17(6):1347-1350.

15. Kelly PJ, Daumas-Duport C, Kispert DB, Kall BA, Scheithauer BW, Illig JJ. Imaging-based stereotaxic serial biopsies in untreated intracranial glial neoplasms. J Neurosurg. 1987;66(6):865-874.

16. Lu S, Ahn D, Johnson G, Law M, Zagzag D, Grossman RI. Diffusion-tensor MR imaging of intracranial neoplasia and associated peritumoral edema: introduction of the tumor infiltration index. Radiology. 2004;232(1): 221-228.

17. Schoenegger K, Oberndorfer S, Wuschitz B, et al. Peritumoral edema on MRI at initial diagnosis: an independent prognostic factor for glioblastoma? Eur J Neurol. 2009;16(7):874-878. 
18. Pope WB, Sayre J, Perlina A, Villablanca JP, Mischel PS, Cloughesy TF. MR imaging correlates of survival in patients with high-grade gliomas. AJNR Am J Neuroradiol. 2005;26(10):2466-2474.

19. Iliadis G, Kotoula V, Chatzisotiriou A, et al. Volumetric and MGMT parameters in glioblastoma patients: survival analysis. BMC Cancer. 2012;12:3.

20. Ramakrishna R, Barber J, Kennedy G, et al. Imaging features of invasion and preoperative and postoperative tumor burden in previously untreated glioblastoma: correlation with survival. Surg Neurol Int. 2010;1:40.

21. Chamberlain MC. Radiographic patterns of relapse in glioblastoma. J Neurooncol. 2011;101(2):319-323.

22. Wallner KE, Galicich JH, Krol G, Arbit E, Malkin MG. Patterns of failure following treatment for glioblastoma multiforme and anaplastic astrocytoma. Int J Radiat Oncol Biol Phys. 1989;16(6):1405-1409.

23. Liang BC, Thornton AF Jr, Sandler HM, Greenberg HS. Malignant astrocytomas: focal tumor recurrence after focal external beam radiation therapy. J Neurosurg. 1991;75(4):559-563.

24. Oppitz U, Maessen D, Zunterer H, Richter S, Flentje M. 3D-recurrencepatterns of glioblastomas after CT-planned postoperative irradiation. Radiother Oncol. 1999;53(1):53-57.

25. Lee SW, Fraass BA, Marsh LH, et al. Patterns of failure following high-dose 3-D conformal radiotherapy for high-grade astrocytomas: a quantitative dosimetric study. Int J Radiat Oncol Biol Phys. 1999;43(1): 79-88.

26. Minniti G, Amelio D, Amichetti M, et al. Patterns of failure and comparison of different target volume delineations in patients with glioblastoma treated with conformal radiotherapy plus concomitant and adjuvant temozolomide. Radiother Oncol. 2010;97(3):377-381.

27. Dobelbower MC, Burnett Iii OL, Nordal RA, et al. Patterns of failure for glioblastoma multiforme following concurrent radiation and temozolomide. J Med Imaging Radiat Oncol. 2011;55(1):77-81.

28. Klein M, Heimans JJ, Aaronson NK, et al. Effect of radiotherapy and other treatment-related factors on mid-term to long-term cognitive sequelae in low-grade gliomas: a comparative study. Lancet. 2002;360(9343): 1361-1368

29. Kleinberg L, Wallner K, Malkin MG. Good performance status of long-term disease-free survivors of intracranial gliomas. Int J Radiat Oncol Biol Phys. 1993;26(1):129-133.

30. Marks JE, Baglan RJ, Prassad SC, Blank WF. Cerebral radionecrosis: incidence and risk in relation to dose, time, fractionation and volume. Int J Radiat Oncol Biol Phys. 1981;7(2):243-252.

31. Swennen MH, Bromberg JE, Witkamp TD, Terhaard CH, Postma TJ, Taphoorn MJ. Delayed radiation toxicity after focal or whole brain radiotherapy for low-grade glioma. J Neurooncol. 2004;66(3):333-339.

32. Jalali R, Mallick I, Dutta D, et al. Factors influencing neurocognitive outcomes in young patients with benign and low-grade brain tumors treated with stereotactic conformal radiotherapy. Int J Radiat Oncol Biol Phys. 2010;77(4):974-979.
33. Walid MS. Prognostic factors for long-term survival after glioblastoma. Perm J. 2008;12(4):45-48.

34. Siker ML, Wang M, Porter K, et al. Age as an independent prognostic factor in patients with glioblastoma: a Radiation Therapy Oncology Group and American College of Surgeons National Cancer Data Base comparison. J Neurooncol. 2011;104(1):351-356.

35. Burton EC, Lamborn KR, Forsyth P, et al. Aberrant p53, mdm2, and proliferation differ in glioblastomas from long-term compared with typical survivors. Clin Cancer Res. 2002;8(1):180-187.

36. Kuhnt D, Becker A, Ganslandt O, Bauer M, Buchfelder M, Nimsky C. Correlation of the extent of tumor volume resection and patient survival in surgery of glioblastoma multiforme with high-field intraoperative MRI guidance. Neuro Oncol. 2011;13(12):1339-1348.

37. Curran WJ Jr, Scott CB, Horton J, et al. Recursive partitioning analysis of prognostic factors in three Radiation Therapy Oncology Group malignant glioma trials. J Natl Cancer Inst. 1993;85(9):704-710.

38. Lacroix M, Abi-Said D, Fourney DR, et al. A multivariate analysis of 416 patients with glioblastoma multiforme: prognosis, extent of resection, and survival. J Neurosurg. 2001;95(2):190-198.

39. Scott JN, Rewcastle NB, Brasher PM, et al. Which glioblastoma multiforme patient will become a long-term survivor? A population-based study. Ann Neurol. 1999;46(2):183-188.

40. Ulutin C, Fayda M, Aksu G, et al. Primary glioblastoma multiforme in younger patients: a single-institution experience. Tumori. 2006;92(5): 407-411.

41. Korshunov A, Sycheva R, Golanov A. The prognostic relevance of molecular alterations in glioblastomas for patients age $<50$ years. Cancer. 2005;104(4):825-832.

42. Simpson JR, Horton J, Scott C, et al. Influence of location and extent of surgical resection on survival of patients with glioblastoma multiforme: results of three consecutive Radiation Therapy Oncology Group (RTOG) clinical trials. Int J Radiat Oncol Biol Phys. 1993;26(2):239-244.

43. DeAngelis LM. Brain tumors. N Engl J Med. 2001;344(2):114-123.

44. Preusser M, Hoeftberger R, Woehrer A, et al. Prognostic value of Ki67 index in anaplastic oligodendroglial tumours - a translational study of the European Organization for Research and Treatment of Cancer Brain Tumor Group. Histopathology. 2012;60(6):885-894.

45. Biernat W. 2000 World Health Organization classification of tumors of the nervous system. Pol J Pathol. 2000;51(3):107-114.

46. Dunn J, Baborie A, Alam F, et al. Extent of MGMT promoter methylation correlates with outcome in glioblastomas given temozolomide and radiotherapy. Br J Cancer. 2009;101(1):124-131.

47. Hegi ME, Diserens AC, Gorlia T, et al. MGMT gene silencing and benefit from temozolomide in glioblastoma. N Engl J Med. 2005;352(10): 997-1003.
OncoTargets and Therapy

\section{Publish your work in this journal}

OncoTargets and Therapy is an international, peer-reviewed, open access journal focusing on the pathological basis of all cancers, potential targets for therapy and treatment protocols employed to improve the management of cancer patients. The journal also focuses on the impact of management programs and new therapeutic agents and protocols on

\section{Dovepress}

patient perspectives such as quality of life, adherence and satisfaction The manuscript management system is completely online and includes a very quick and fair peer-review system, which is all easy to use. Visit http://www.dovepress.com/testimonials.php to read real quotes from published authors. 